\title{
Preparation of Dibenzazepine-containing Polymers and Use as Fluorescent Functional Additives for Estimating Plastic Blend
}

\author{
Hideki HAYASHI
}

Nagoya Municipal Industrial Research Institute, 3-4-41 Rokuban, Atsuta, Nagoya 456-0058, Japan

\begin{abstract}
Dibenzazepine-containing polymers were prepared by various polymerization methods and the effects of adding the obtained polymers to plastics were investigated. Dibenzazepine homopolymers were prepared by the oxidative polymerization of commercially available dibenzazepine derivatives and by the dehalogenative polymerization of corresponding dibromodibenzazepines. Dibenzazepine copolymers were prepared from dibromodibenzazepines. The addition of polymers to plastics afforded fluorescent function to them. The fluorescent behavior of a blend composite depended not only on the chemical structure of the polymers, but also on the plastic used as the matrix. The effects of adding dibenzazepine-containing polymer in plastic blends were investigated. When the compatibility of the plastic blend was higher, the fluorescence $\lambda_{\max }$ of the blend became shorter. This suggested that the method using a dibenzazepinecontaining polymer is adaptive for estimating of the compatibility of the blend composite by the simple method.
\end{abstract}

Keywords Dibenzazepine-containing polymer, fluorescent additive, plastic blend, estimation of compatibility

(Received August 30, 2019; Accepted November 11, 2019; Advance Publication Released Online by J-STAGE November 22, 2019)

1 Introduction

2 Preparation of Dibenzazepine Homopolymers and Their Use as Functional Additives for Plastics

2.1 Preparation by oxidative polymerization

2.2 Preparation by dehalogenative polymerization

3 Preparation of Dibenzazepine Copolymers and Their Use as Functional Additives for Plastics

3.1 Preparation of dibenzazepine copolymers

3.2 Using PAzep-Fls as functional additives
7 Supporting Information 24

\section{Introduction}

Polymers containing diphenylamine a with bridging structure (Fig. 1) at their main chain, such as $\mathrm{O},{ }^{1} \mathrm{~S},{ }^{2-4} \mathrm{SO},{ }^{3,4} \mathrm{SO}_{2},{ }^{4}$ $-\mathrm{CH}_{2} \mathrm{CH}_{2^{-}},{ }^{5,6}$ and $-\mathrm{SiR}_{2-},{ }^{7-10}$ often show interesting optical and

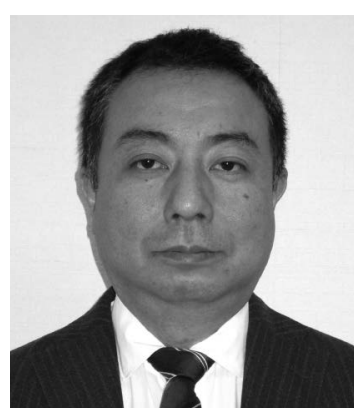

Hideki HaYaSHI received Ph.D. degree in engineering from Tokyo Institute of Technology in 1998. He worked at Japan Chemical Innovation Institute (1998 2001) and Nagoya Municipal Industrial Research Institute (2001 -). He became a senior researcher in 2014. His current research interest is preparation, chemistry and application of $\pi$-conjugated materials.

E-mail: hayashi.hideki@nmiri.city.nagoya.jp

electrochemical properties, and thus have been researched. Our group has reported about $\mathrm{Si}$ unit-bridged diphenylaminecontaining polymers as electric devices, ${ }^{11-17}$ conjugation with DNA $^{11,18,19}$ and carbonnanotubes..$^{11,20}$

On the other hand, diphenylamines with a bridging structure are expected to have thermal stability. ${ }^{7}$ Therefore, these compounds were selected as additives for plastics because these types of polymers seem to be useful as functional additives in plastics, by using a thermal melting process usually used in processing plastics. Usually, plastics are functionalized by additives. Therefore, many additives for plastics have been widely researched.

Poly(lactic acid) (PLA, Fig. 2) was used as the plastic. PLA is a synthetic biopolymer derived from biomass through bioconversion and polymerization, ${ }^{21,22}$ and therefore the modification and functionalization of PLA are widely researched. $^{23-39}$ Our group has developed additives for PLA, such as a nucleating agent, $, 00,41$ a layered silicate, ${ }^{42}$ and a chemically modified poly(3-hydroxyalkanoate). ${ }^{43}$ On the other hand, to make PLA with fluorescence function, we added 


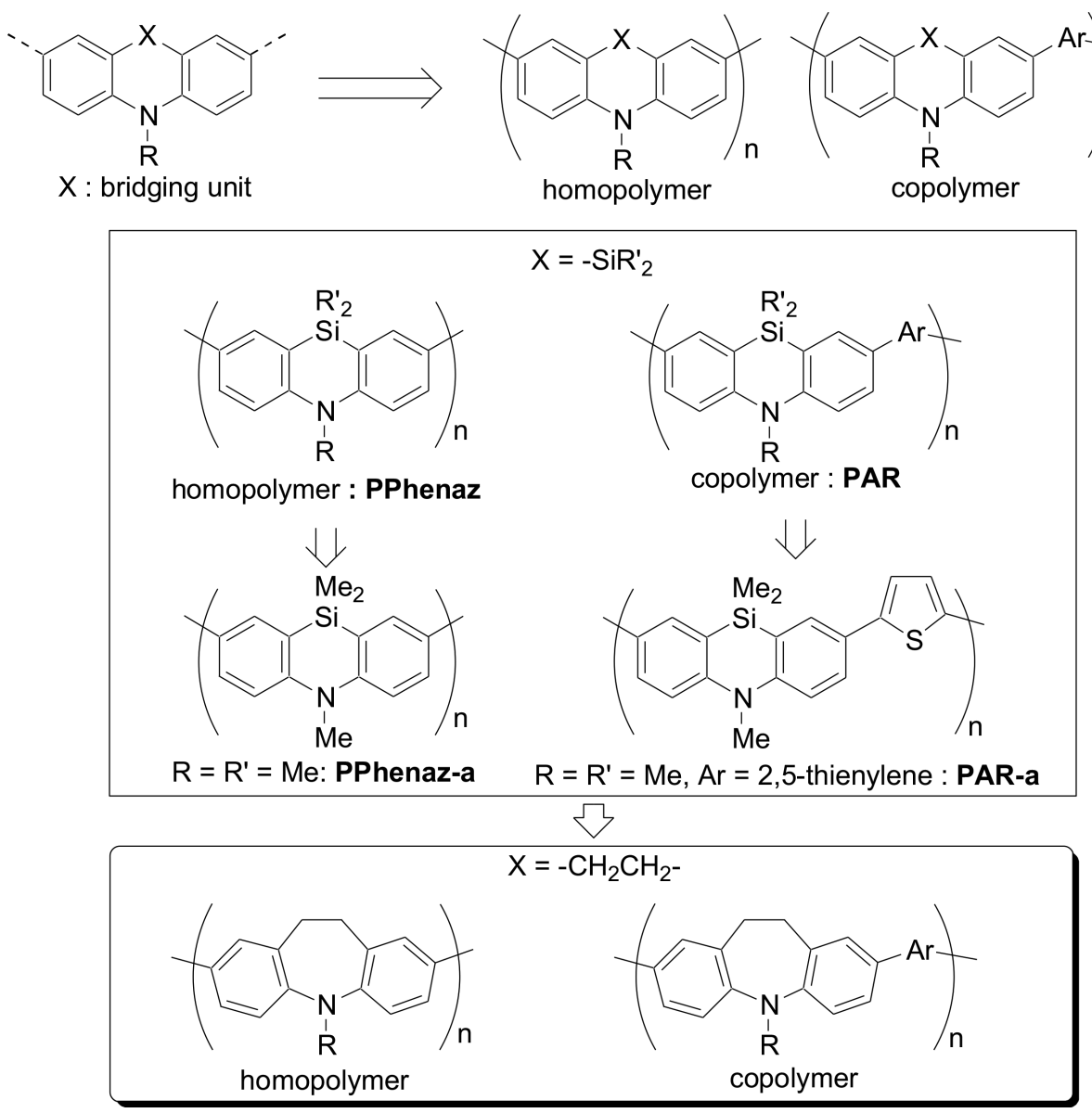

Fig. 1 Chemical structure of poly(diphenylamine)s with a bridged structure and relative polymers.

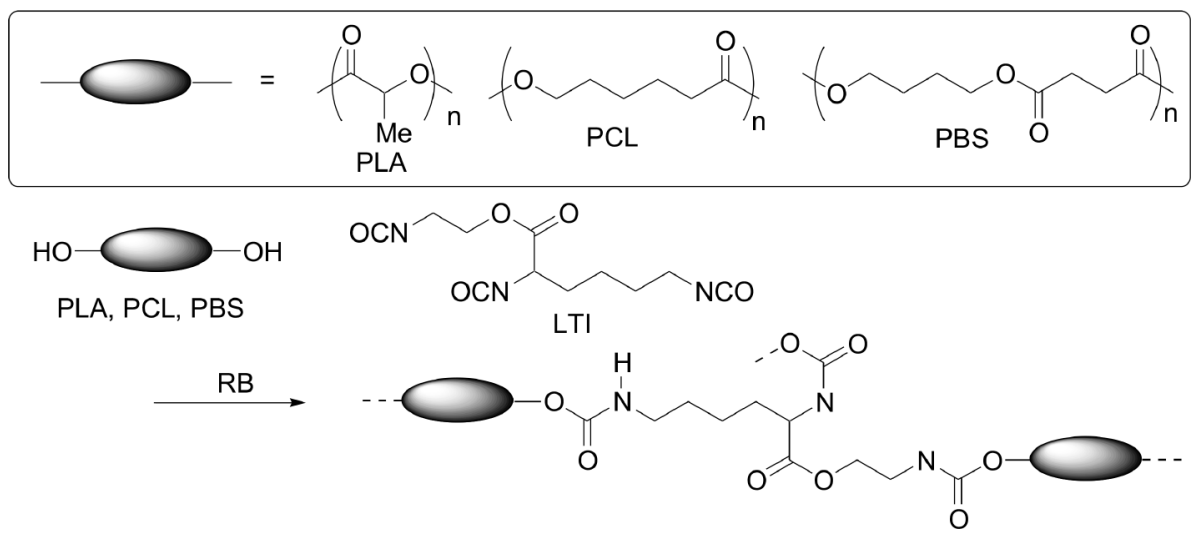

Fig. 2 Chemical structure of plastics and the reaction scheme of the reactive blend (RB).

polymers containing diphenylamine with a monosilane-bridging unit (PPhenaz, Fig. 1). 12,44,45

However, it needs several steps to prepare phenazasilinecontaining polymers because phenazasiline unit compounds are not usually purchased; therefore, simplification of the preparation process of the polymer is needed. In the present work, dibenzazepine was selected as diphenylamine with a bridged structure because some dibenzazepine derivatives are commercially available products. ${ }^{46-48}$ Thus, investigating the preparation of dibenzazepine-containing polymers and the use of polymers as the functional additives for plastics are carried out.

By the way, plastics are sometimes used as blend composites of two or more kinds of plastics. For example, PLA's mechanical properties have been improved by a reactive blend (RB), which is a method of adding multifunctional isocyanates, such as lysine triisocyanate (LTI, Fig. 2) as compatibilizers. The present method is the addition of LTI as a reagent to the terminal hydroxyl group of polyesters, such as PLA, poly( $\varepsilon$ caprolactone) (PCL, Fig. 2), ${ }^{49}$ and poly(butylenesuccinate) 
(PBS, Fig. 2)..$^{50}$ It is usually necessary for estimating compatibility to use difficult techniques, such as using AFM and measuring the physical properties. These estimation methods sometimes need a high level or complicated techniques or breaking samples; consequently, a blend estimation by a simple and easy method is needed. Therefore, the fluorometry was chosen as the estimation method of compatibility of plastic blends, because this is an easy and nondestructive method. The method for estimating compatibility by measuring fluorescence spectra has already been reported, ${ }^{12}$ but that method needed two kinds of fluorescent additives (PPhenaz-a and PAR-a, Fig. 1). However, further simplication is needed. In this paper, it is described that the investigation of a simple method for estimating the compatibility of a plastic blend, by using dibenzazepinecontaining polymers as a fluorescent probe, as in Fig. 3.

\section{Preparation of Dibenzazepine Homopolymers and Their Use as Functional Additives for Plastics}

\subsection{Preparation by oxidative polymerization ${ }^{51}$}

As described above, dibenzazepine derivatives are expected as properties of diphenylamine, and some $N$-substituted dibenzazepine derivatives show physiological activity. Therefore, it should be easier to develop dibenzazepinecontaining polymers than phenazasiline-containing polymers because some dibenzazepine derivatives are commercially

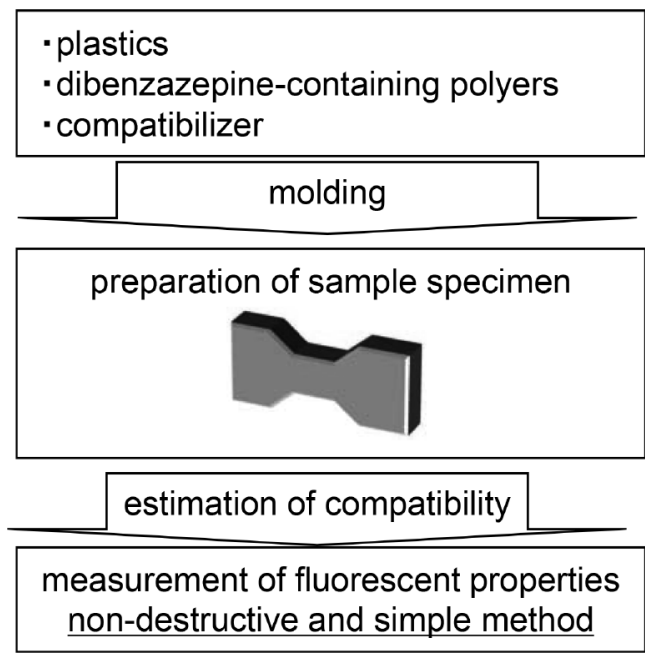

Fig. 3 Scheme of estimating the compatibility of plastic blends in this paper. available products.

First, the oxidative polymerization of dibenzazepine derivatives, which are commercial products, was carried out. Oxidative polymerization is known to be one of the most convenient polymerization methods, ${ }^{52,53}$ and poly(arylamine) type polymers were prepared by this method. ${ }^{13,54,55}$ Accordingly, PAzep-as were prepared by the oxidative polymerization of corresponding Azeps, as shown in Fig. 4, by referring to the literature. $^{13}$ The prepared polymers are shown in Table 1. Generally, polymers prepared from oxidative polymerization were dedoped by dedoping reagents, such as hydrazine monohydrate (for the preparation of poly(diphenylamine) $\mathrm{s}^{13}$ ) and methanol (for the preparation of poly(thiophene) $\mathrm{s}^{53}$ ). In this polymerization, hydrazine monohydrate was used as the dedoping reagent. Therefore, polymers with an $\mathrm{N}$-aminoalkyl substituent were obtained by neutralizing their hydrochloride unit because hydrazine monohydrate was used as a dedoping reagent, and it worked not only as a dedoping reagent, but also as a base.

The fluorescence intensities of PAzep-a-added plastics were stronger than that of raw plastic, suggesting that PAzep-as are effective as fluorescent additives.

\subsection{Preparation by dehalogenative polymerization ${ }^{56,57}$}

Oxidative polymerization is a convenient method for the preparation of polymers, but sometimes the juncture of the polymer was not controlled. Therefore, dehalogenative polymerization was adopted, because this method gives structurally well-defined $\pi$-conjugated polymers. ${ }^{58,59}$ In this way, dibromodibenzazepine derivatives (Azep-Brs) were prepared and polycondensations of Azep-Brs by Ni complexes ${ }^{58}$ were carried out to obtain PAzep-bs, as shown in Fig. 5, and the prepared PAzep-bs are given in Table 2.

The absorption $\lambda_{\max }$ of a polymer with $\mathrm{H}$ on the side chain (PAzep-b1) is longer than that of $N$-alkyl substituted polymers, especially prepared by dehalogenative polymerization. This result was almost the same as that of poly(phenazasiline) ${ }^{15}$ and poly(diphenylamine) ${ }^{60}$ However, the absorption $\lambda_{\max }$ of PAzepas was different from that of PAzep-bs, suggesting that the optical properties of PAzeps depend on their preparation method.

Table 1 Prepared PAzeps by oxidative polymerization and used monomers

\begin{tabular}{ccc}
\hline Polymer & $\mathrm{R}^{1}$ & \multicolumn{1}{c}{$\mathrm{R}$} \\
\hline PAzep-a1 & $\mathrm{H}$ & $\mathrm{H}$ \\
PAzep-a2 & $-\mathrm{CH}_{2} \mathrm{CH}_{2} \mathrm{CH}_{2} \mathrm{NHMe}_{2} \mathrm{Cl}$ & $-\mathrm{CH}_{2} \mathrm{CH}_{2} \mathrm{CH}_{2} \mathrm{NMe}_{2}$ \\
PAzep-a3 & $-\mathrm{CH}_{2} \mathrm{CH}_{2} \mathrm{CH}_{2} \mathrm{NH}_{2} \mathrm{MeCl}$ & $-\mathrm{CH}_{2} \mathrm{CH}_{2} \mathrm{CH}_{2} \mathrm{NHMe}$ \\
\hline
\end{tabular}

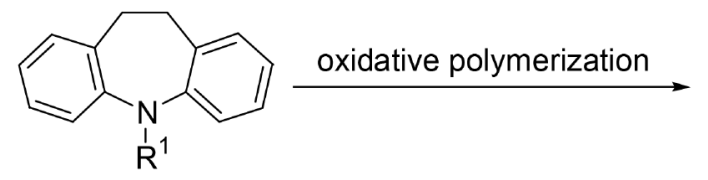

Azep-1: $\mathrm{R}^{1}=\mathrm{H}$

PAzep-a1: $\mathrm{R}=\mathrm{H}$

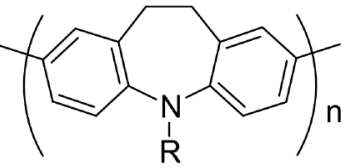

PAzep

Azep-2-HCl: $\mathrm{R}^{1}=\mathrm{CH}_{2} \mathrm{CH}_{2} \mathrm{CH}_{2} \mathrm{~N}^{+} \mathrm{Me}_{2} \mathrm{HCl}^{-}$

PAzep-a2: $\mathrm{R}=\mathrm{CH}_{2} \mathrm{CH}_{2} \mathrm{CH}_{2} \mathrm{NMe}_{2}$

Azep-3-HCl: $\mathrm{R}^{1}=\mathrm{CH}_{2} \mathrm{CH}_{2} \mathrm{CH}_{2} \mathrm{~N}^{+} \mathrm{H}_{2} \mathrm{MeCl}^{-}$

PAzep-a3: $\mathrm{R}=\mathrm{CH}_{2} \mathrm{CH}_{2} \mathrm{CH}_{2} \mathrm{NHMe}$

Fig. 4 Oxidative polymerization scheme of Azeps. 
<smiles>[R]N1c2ccc(Br)cc2CCc2cc(I)ccc21</smiles>

Azep-Br-1: $\mathrm{R}=\mathrm{H}$

Azep-Br-2: $\mathrm{R}=-\mathrm{CH}_{2} \mathrm{CH}_{2} \mathrm{CH}_{2} \mathrm{NMe}_{2}$

Azep-Br-4: $\mathrm{R}=-\mathrm{CH}_{2} \mathrm{CH}_{2} \mathrm{CH}_{2} \mathrm{NMeBoC}$

Azep-Br-5: $\mathrm{R}=\mathrm{Me}$

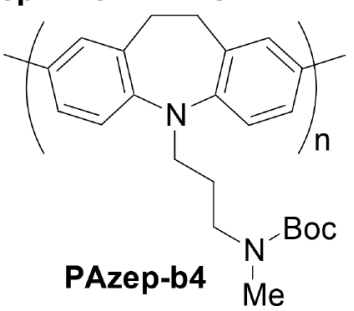

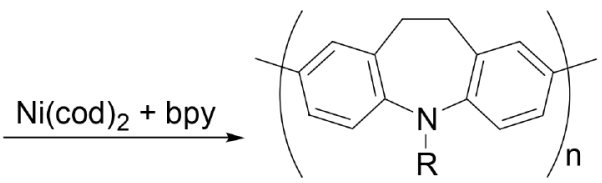

PAzep-b1: $\mathrm{R}=\mathrm{H}$

PAzep-b2: $\mathrm{R}=-\mathrm{CH}_{2} \mathrm{CH}_{2} \mathrm{CH}_{2} \mathrm{NMe}_{2}$

PAzep-b4: $\mathrm{R}=-\mathrm{CH}_{2} \mathrm{CH}_{2} \mathrm{CH}_{2} \mathrm{NMeBoC}$

PAzep-b5: $\mathrm{R}=\mathrm{Me}$

Fig. 5 Preparation scheme of PAzep-bs.

Table 2 Optical properties of PAzep-bs

\begin{tabular}{|c|c|c|c|c|}
\hline Polymer & $\mathrm{X}$ & $\mathrm{R}$ & $\begin{array}{c}\text { Absorption } \\
\lambda_{\max } / \mathrm{nm}^{\mathrm{a}}\end{array}$ & $\begin{array}{c}\text { Fluorescence } \\
\lambda_{\max } / \mathrm{nm}^{\mathrm{a}, \mathrm{b}}\end{array}$ \\
\hline PAzep-b1 & $-\mathrm{CH}_{2} \mathrm{CH}_{2-}$ & $\mathrm{H}$ & $344^{c}$ & $409^{c}$ \\
\hline PAzep-b2 & $-\mathrm{CH}_{2} \mathrm{CH}_{2-}$ & $-\mathrm{CH}_{2} \mathrm{CH}_{2} \mathrm{CH}_{2} \mathrm{NMe}_{2}$ & 321 & 411 \\
\hline PAzep-b3 & $-\mathrm{CH}_{2} \mathrm{CH}_{2-}$ & $-\mathrm{CH}_{2} \mathrm{CH}_{2} \mathrm{CH}_{2} \mathrm{NHMe}$ & $321^{\mathrm{c}}$ & $406^{c}$ \\
\hline PAzep-b4 & $-\mathrm{CH}_{2} \mathrm{CH}_{2-}$ & $-\mathrm{CH}_{2} \mathrm{CH}_{2} \mathrm{CH}_{2} \mathrm{NMeBoc}$ & 321 & 404 \\
\hline PAzep-b5 & $-\mathrm{CH}_{2} \mathrm{CH}_{2-}$ & $\mathrm{Me}$ & $332^{\mathrm{c}}$ & $395^{\mathrm{c}}$ \\
\hline
\end{tabular}

a. $\mathrm{CHCl}_{3}$ solution.

b. Excited at absorption $\lambda_{\max }$.

c. $\mathrm{CHCl}_{3}$ soluble part.<smiles>[X]c1cc(C(C)C)ccc1N(C)c1ccc(C)cc1C</smiles>

$$
\begin{aligned}
& \text { X }=-\mathrm{SiMe}_{2}-: \text { PPhenaz-Me } \\
& \text { X=-SiMe } \mathrm{SiMe}_{2}-: \text { PDSiAzep } \\
& \text { X }=-\mathrm{SiMe}_{2} \mathrm{OSiMe}_{2}-\text { PSiO }
\end{aligned}
$$

Fig. 6 Polymers for comparing optical properties.

The optical properties of poly( $N$-alkyldiphenylamine)s with a bridged structure were compared. A comparison of the polymers' optical properties with PAzep-b5 is shown in Fig. $6 .{ }^{17}$ The absorption $\lambda_{\max }$ of the polymer was in the order $\mathrm{Si}>\mathrm{CH}_{2} \mathrm{CH}_{2}>\mathrm{SiOSi}>\mathrm{SiSi}$, as shown in Table 3. To investigate the effect of the structure, the X-ray data of the monomers were compared. ${ }^{17,61}$ The sums of the angles around the $\mathrm{N}$ of the diphenylamine unit are also given in Table 3 . As shown in the table, the sums of the bond angles around the $\mathrm{N}$ atom of the corresponding monomers were in the order PPhenaz-a $>$ PAzep-5b $>$ PSiO $>$ PDSiAzep. These results suggest that the absorption $\lambda_{\max }$ of this type of polymers also depends on the flatness around the $\mathrm{N}$ atom of the monomer unit.

The addition of PAzep-b to plastic gave the same fluorescence
Table 3 Comparison between the absorption $\lambda_{\max }$ of polymers and the flatness of the $\mathrm{N}$ atom of corresponding monomers

\begin{tabular}{llcc}
\hline \multicolumn{1}{c}{ Polymer } & \multicolumn{1}{c}{$\mathrm{X}$} & $\begin{array}{c}\text { Absorption } \\
\lambda_{\max } / \mathrm{nm}^{\mathrm{a}}\end{array}$ & $\begin{array}{c}\text { Sum of bond } \\
\text { angles around the } \\
\mathrm{N} \text { atom of relative } \\
\text { monomeric } \\
\text { compound/deg }\end{array}$ \\
\hline PAzep-b5 & $-\mathrm{CH}_{2} \mathrm{CH}_{2-}$ & $332^{\mathrm{b}}$ & $351.5^{\mathrm{c}}$ \\
PPhenaz-Me & $-\mathrm{SiMe}_{2}-$ & $361^{\mathrm{b}, \mathrm{d}}$ & $358.5^{\mathrm{d}}$ \\
PDSiAzep & $-\mathrm{SiMe}_{2} \mathrm{SiMe}_{2-}$ & $274^{\mathrm{d}}$ & $337.8^{\mathrm{d}}$ \\
PSiO & $-\mathrm{SiMe}_{2} \mathrm{OSiMe}-$ & $315^{\mathrm{d}}$ & $341.2^{\mathrm{d}}$ \\
\hline
\end{tabular}

a. $\mathrm{CHCl}_{3}$ solution.

b. $\mathrm{CHCl}_{3}$ soluble part.

c. Data of Azep-2-HCl from Ref. 61. Two types of crystals were observed. The angle shown in the table was the average value (the angles were $354.7^{\circ}$ and $348.3^{\circ}$ ).

d. Data from Ref. 17.

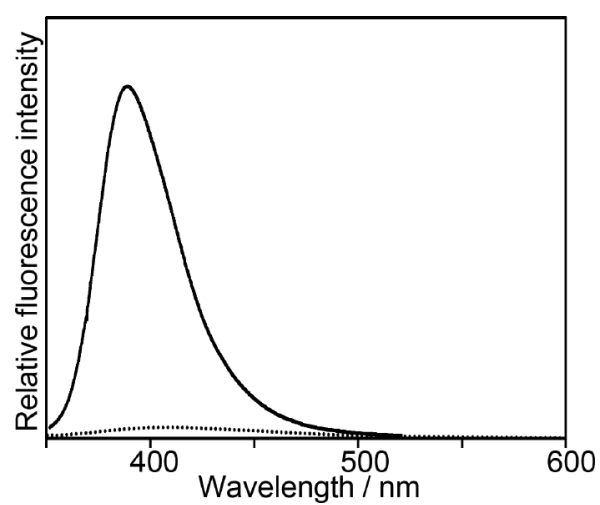

Fig. 7 Fluorescence spectra of PLA with 0.005 phr PAzep-b5 (solid line) and without PAzep-b5 (dotted line).

as by the addition of PAzep-a. The fluorescence spectra of PAzep-b-added PLA and raw PLA are shown in Fig. 7. The efficiency of the fluorescent additive of PAzep-b seems to be higher than that of PAzep-a. The change of this efficiency 


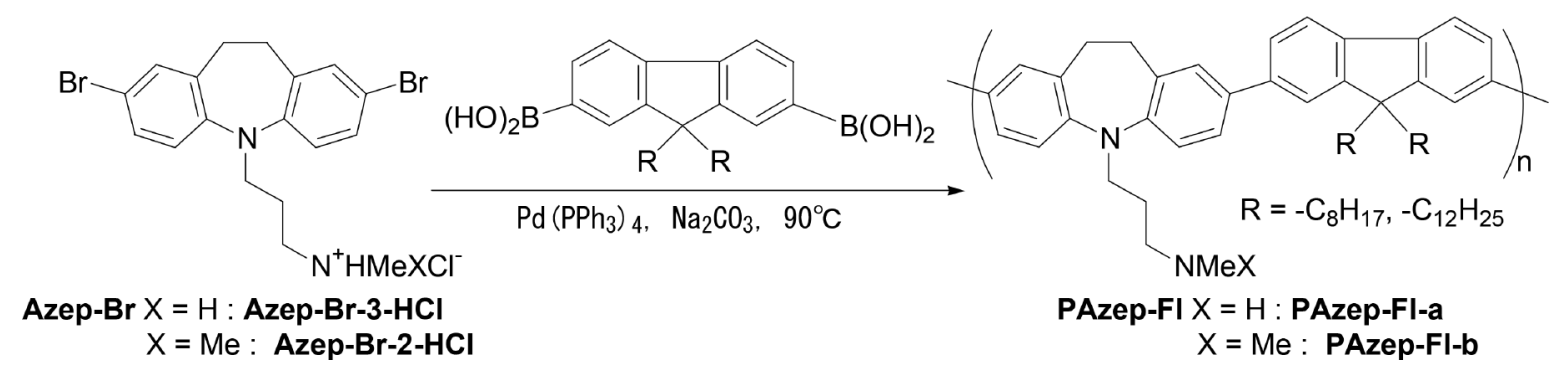

Fig. 8 Preparation scheme of PAzep-Fls.

Table 4 Prepared PAzep-Fls and optical properties of polymers

\begin{tabular}{lcccccc}
\hline \multicolumn{1}{c}{ Polymer } & $R^{\prime}$ & $R^{\prime \prime}$ & $\begin{array}{c}\mathrm{CHCl}_{3} \text { solution } \\
\text { absorption } \\
\lambda_{\max } / \mathrm{nm}^{\mathrm{a}}\end{array}$ & $\begin{array}{c}\mathrm{CHCl}_{3} \text { solution } \\
\text { fluorescence } \\
\lambda_{\max } / \mathrm{nm}^{\mathrm{a}, \mathrm{b}}\end{array}$ & $\begin{array}{c}\text { Cast Film } \\
\text { fluorescence } \\
\lambda_{\max } / \mathrm{nm}^{\mathrm{a}, \mathrm{c}}\end{array}$ \\
\hline PAzep-Fl-a8 & $\mathrm{H}$ & $-\mathrm{C}_{8} \mathrm{H}_{17}$ & 352 & 417 & 439 \\
PAzep-Fl-a12 & $\mathrm{H}$ & $-\mathrm{C}_{12} \mathrm{H}_{25}$ & 347 & 418 & 436 \\
PAzep-Fl-b8 & $\mathrm{Me}$ & $-\mathrm{C}_{8} \mathrm{H}_{17}$ & 346 & 420 & 434 \\
PAzep-Fl-b12 & $\mathrm{Me}$ & $-\mathrm{C}_{12} \mathrm{H}_{25}$ & 353 & 426 & 438 \\
\hline
\end{tabular}

a. $\mathrm{CHCl}_{3}$ solution.

b. Excited at absorption $\lambda_{\max }$.

c. Cast from $\mathrm{CHCl}_{3}$.

seems to because of the following reasons. The first is that there are no structure defects in PAzep-b. The other is that PAzep-a was dedoped by the dedoping reagent, but sometimes a small amount of dopant remained in the polymers, so the dopant reduced the efficiency as the fluorescence additive.

\section{Preparation of Dibenzazepine Copolymers and Their Use as Functional Additives for Plastics}

\section{3·1 Preparation of dibenzazepine copolymers ${ }^{62,63}$}

Azep-Brs can be useful not only as a monomer of dibenzazepine homopolymers, but also as a monomer of dibenzazepine copolymers, because many copolymers have been prepared from corresponding dibromide by cross-coupling reactions. ${ }^{58}$ Therefore, the preparation of dibenzazepinecontaining copolymers (PAzep-FI) by using Azep-Br as monomers, and their use as a functional additive for plastics were investigated.

The preparation scheme of PAzep-Fl is shown in Fig. 8. PAzep-Fls were prepared from the Suzuki-Miyaura coupling reaction $^{9,58}$ of corresponding dibromodibenzazepines (Azep-2Br-HCl and Azep-3-Br-HCl) and 9,9-dialkylfluorene-2,7diboronic acid; the resulting polymers are shown in Table 4. The obtained PAzep-Fl was soluble in the usual organic solvents, such as chloroform and THF. These IR and NMR spectra suggest that most of dibromodibenzazepines' (Azep-2Br-HCl and Azep-3-Br-HCl) ammonium unit was converted to amine unit by the polymerization process, because the polymerizations were carried out under the basic condition. Neutralization of the polymers is also suggested by elemental analysis of the $\mathrm{Cl}$ content of the polymers.

The optical properties of PAzep-Fls are given in Table 4. The absorption and fluorescence $\lambda_{\max }$ of the solution suggest that long alkyl chains do not affect their optical properties in the same way as PAzep-b ${ }^{57}$ and PPhenaz. ${ }^{15}$ The fluorescence $\lambda_{\max }$ of the cast film was longer than that of the $\mathrm{CHCl}_{3}$ solution.
Table 5 Prepared PLA/PCL blend composites and their fluorescence $\lambda_{\max }$

\begin{tabular}{lrrlll}
\hline Sample & $\begin{array}{r}\text { PLA, } \\
\text { wt\% }\end{array}$ & $\begin{array}{r}\text { PCL, } \\
\text { wt\% }\end{array}$ & Added PAzep-Fl ${ }^{\mathrm{a}}$ & $\begin{array}{c}\text { LTI/ } \\
\text { phr }\end{array}$ & $\begin{array}{c}\text { Fluorescence } \\
\lambda_{\text {max }} / \mathrm{nm}^{\mathrm{b}}\end{array}$ \\
\hline PLA-a & 100 & 0 & PAzep-Fl-a (0.01) & 0 & 412 \\
PLA-aT & 100 & 0 & PAzep-Fl-a (0.01) & 0.5 & 405 \\
PCL-a & 0 & 100 & PAzep-Fl-a (0.01) & 0 & 435 \\
PCL-aT & 0 & 100 & PAzep-Fl-a (0.01) & 0.5 & 415 \\
PLA-b & 100 & 0 & PAzep-Fl-b (0.01) & 0 & 421 \\
PLA-bT & 100 & 0 & PAzep-Fl-b (0.01) & 0.5 & 412 \\
PCL-b & 0 & 100 & PAzep-Fl-b (0.01) & 0 & 438 \\
PCL-bT & 0 & 100 & PAzep-Fl-b (0.01) & 0.5 & 429 \\
\hline
\end{tabular}

a. The figures in parenthesis show the added ratio (phr).

b. Excited at absorption $\lambda_{\text {max }}$ of PAzep-Fl in $\mathrm{CHCl}_{3}$.

\subsection{Using PAzep-Fls as functional additives ${ }^{63,64}$}

For use as functional additives, PAzep-Fls were added to plastics; the blend ratio is given in Table 5. PLA and PCL were chosen as the plastics because PCL was used for modifying PLA. ${ }^{49}$ As shown in Table 5 and Fig. 9, the fluorescence $\lambda_{\max }$ of PAzep-Fl added PLA was almost the same as PAzep-Fl in $\mathrm{CHCl}_{3}$. This suggests that PAzep-Fl has good dispersibility in PLA, similar to PPhenaz. ${ }^{44,45}$ On the other hand, the fluorescence $\lambda_{\max }$ of PAzep-Fl-containing PCL was almost in the same region as that of PAzep-Fl cast film, suggesting that $\pi$-stacking by aggregation ${ }^{65-67}$ of PAzep-Fl occurred in PCL because of its poor dispersibility in PCL. These results suggest that the compatibility of PAzep-Fl to the plastic depended on the plastic used, as shown in Fig. 10. This type of aggregation behavior of the $\pi$-conjugated functional additive was observed when PCL was used as a matrix plastic. ${ }^{68}$

\section{Estimating the Compatibility of a Plastic Blend by Measuring the Fluorescence Spectra}

\subsection{Investigation of effect of addition of LTI $^{63,64}$}

As described above, PAzep-FI was effective as a fluorescent additive, and plastics are sometimes used as blend composites of two or more kinds of plastics, like in Fig. 2. The present method involves the addition of LTI as a reagent to the terminal hydroxyl group of polyesters. To investigate the effect of PAzep-Fl on the blend composite, PAzep-Fl was blended to a PLA/PCL/LTI blend. ${ }^{49}$ First, the effect of adding PAzep-Fl to one kind of plastic/LTI blend was investigated. The blend ratio and fluorescence $\lambda_{\max }$ of the blend composites are given in Table 5. As shown in Fig. 11 and Table 5, the fluorescence $\lambda_{\max }$ became shorter upon the addition of LTI. This effect seems to be explicable as follows: in PAzep-Fl-a, which has a reactive 
(a) PAzep-FI-a

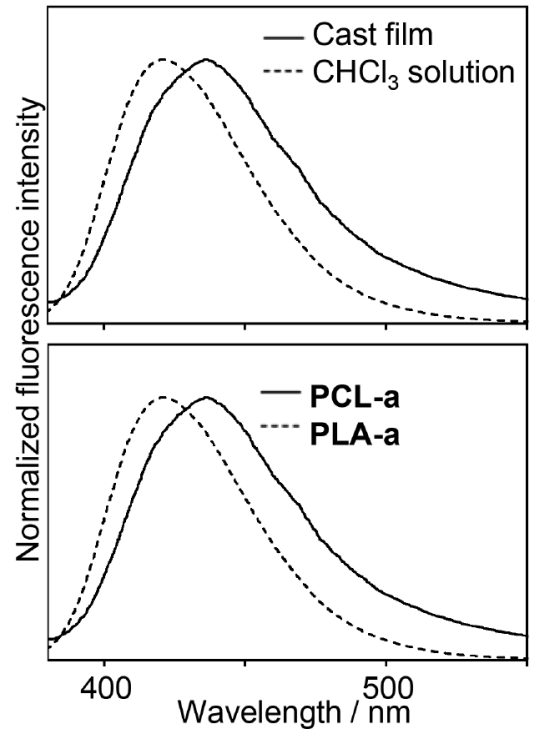

(b) PAzep-FI-b

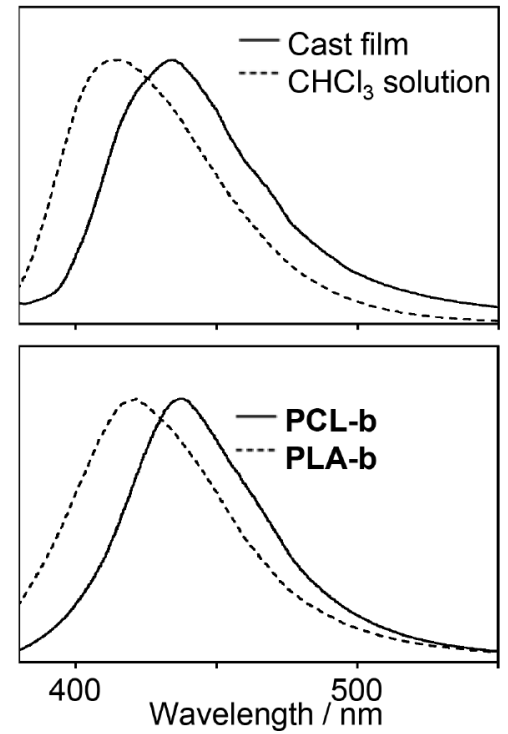

Fig. 9 Comparison of the fluorescence spectra of PAzep-Fls.
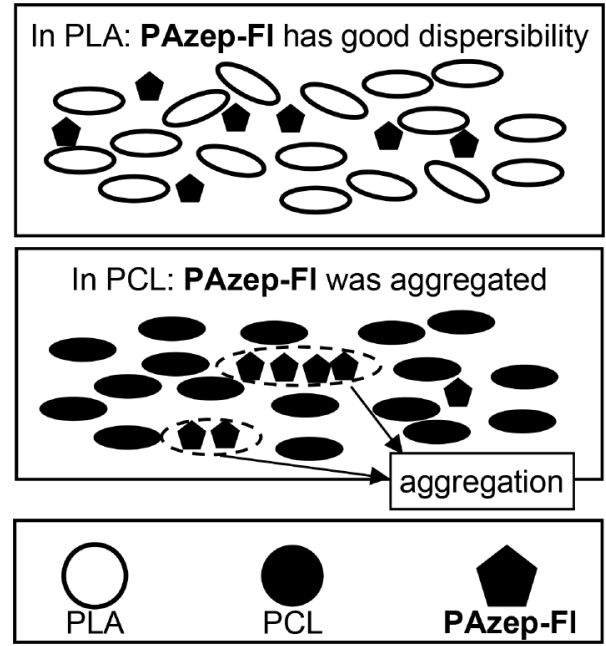

Fig. 10 Supposed dispersion scheme of PAzep-Fls in PLA or PCL.

secondary amine unit, the distance between the PAzep-Fl molecules seems to become longer because of chemical bond formation of the NH unit of PAzep-Fl-a and the plastics via LTI. The reason for this is that a network type structure was formed by the reaction of polyester and LTI, as shown in Fig. 12(a). This network structure causes the distance of PAzep-Fl molecules to increase compared with the absence of LTI. Therefore, a larger effect of the addition of LTI was observed when PCL was used as a matrix plastic than when PLA was used.

However, when PAzep-Fl-b, which has a non-reactive tertiary amine on its side chain, was used as a fluorescent additive, the effect of adding LTI was lower. This can be explained as follows. As shown in Fig. 12(b), when LTI was added to the plastic/PAzep-Fl-b blend composite, a network-type structure was formed by the reaction of polyester and LTI. This network structure seems to cause the distance of PAzep-Fl-b molecules to increase compared with the absence of LTI, because PAzepFl-b seems to be incorporated into the plastic/LTI network.
Therefore, the addition of LTI causes a shorter fluorescence $\lambda_{\max }$. However, the difference in the wavelength by the addition of LTI was smaller than that of PAzep-Fl-a as a fluorescent additive, because chemical bond formation between PAzep-Fl-b and PCL did not occur. These were also suggested by measuring the molecular weight of the blend composites and their impact strength.

\subsection{Estimating the compatibility of a plastic blend by measuring the fluorescence spectra ${ }^{63,64}$}

Next, the effect on the fluorescent properties of adding PAzepFl to PLA/PCL/LTI blend composites was investigated. The blend ratios and their fluorescent properties are given in Table 6 and typical fluorescence spectra are shown in Fig. 13, and the others are shown in Figs. S1 and S2. The fluorescence $\lambda_{\max }$ of the plastic vs. PLA ratio was plotted as shown in Fig. 14. The solid line and dotted line indicate the fluorescence $\lambda_{\max }$ of PAzep-Fl-a and PAzep-Fl-b, respectively. When the PLA/PCL blend ratio was the same, the fluorescence $\lambda_{\max }$ of LTI-containing plastics was shorter than that of the plastics in the absence of LTI. This result suggests that PAzep-Fl was effective as a probe for estimating the compatibility, whereas PPhenaz without PAR was not effective for estimating the compatibility of a plastic blend. ${ }^{45}$

The fluorescent properties of PAzep-FI were also measured in solution; the results are shown as $\times$ in Fig. 14, and their fluorescence spectra are shown in Fig. S3. As described above, the fluorescence $\lambda_{\max }$ of a $\mathrm{CHCl}_{3}$ solution of PAzep-Fl was almost the same wavelength as that of PLA-a and PLA-b, suggesting that PAzep-Fl has good compatibility to PLA. However, the fluorescence $\lambda_{\max }$ of a methanol solution of PAzep-Fl was almost the same wavelength as PAzep-Fl cast film as well as PCL-a and PCL-b. This suggests that PAzepFls were aggregated in PCL as in methanol, such as poly(alkylthiophene) and poly(alkylthiazole). ${ }^{66}$ In addition, we also measured the fluorescence spectra in a mixed solvent of chloroform and methanol with various weight ratios. As shown in the figure, the fluorescence $\lambda_{\max }$ in a mixed solvent was almost the same wavelength as the fluorescence $\lambda_{\max }$ in PLA/PCL blend without using LTI. This result suggests that the 
(a) Composite with PAzep-FI-a

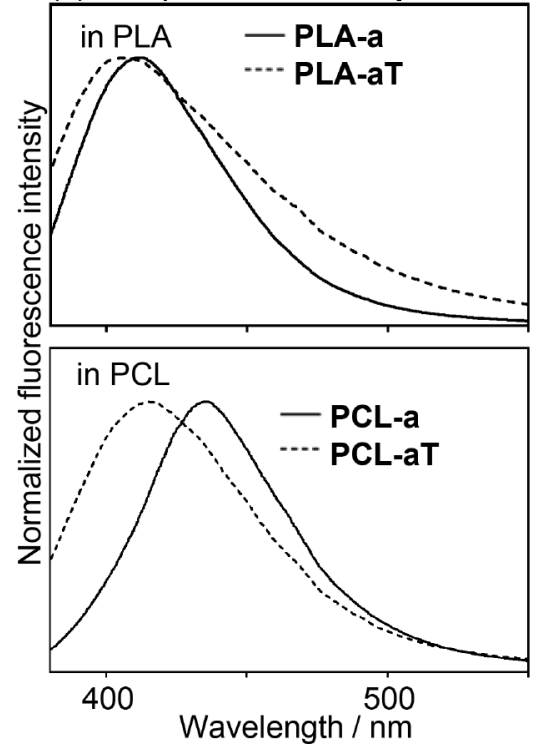

(b) Composite with PAzep-FI-b
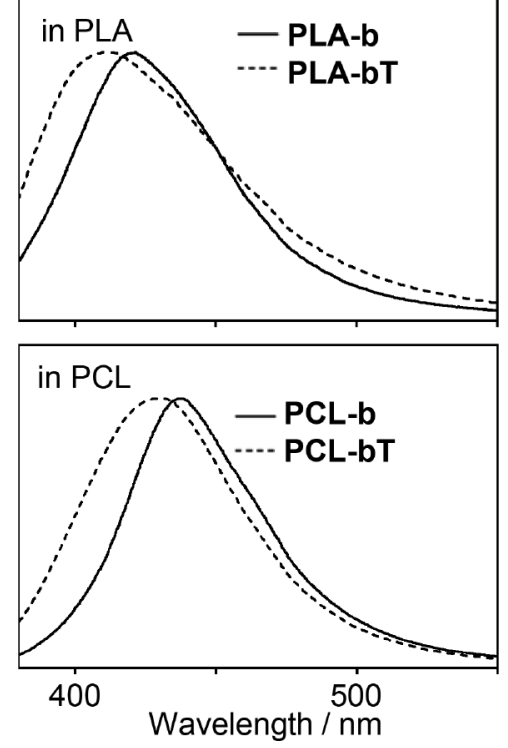

Fig. 11 Fluorescence spectra of PAzep-Fls in PLA or PCL and the effects of adding LTI.

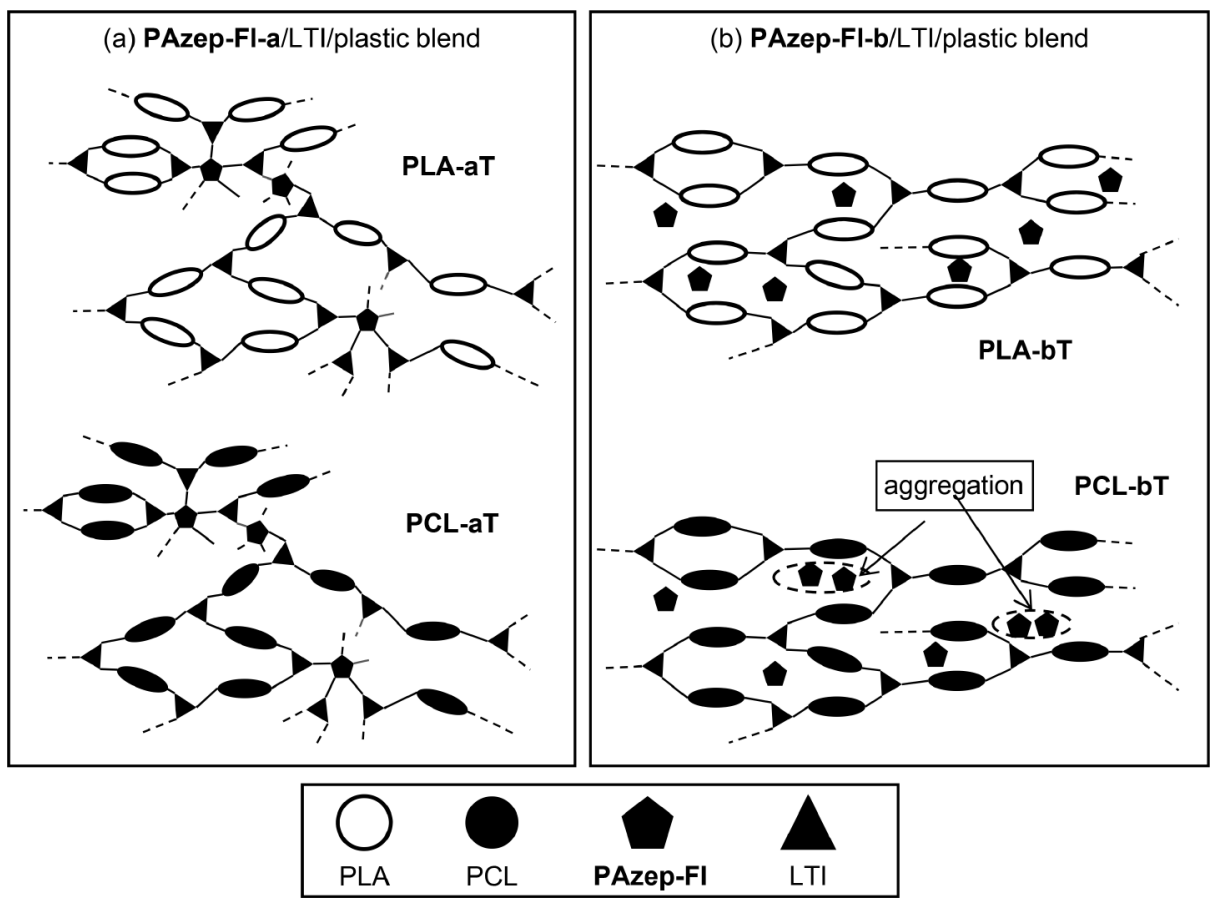

Fig. 12 Supposed dispersion of PAzep-Fls in PLA or PCL with LTI.

measurement of solvatochromism of a fluorescent additive is an easy way to check whether the additive is suitable as a reagent for estimating the compatibility of a plastic blend.

\section{Conclusions}

Prepared dibenzazepine-containing polymers afford fluorescent properties to plastics. Furthermore, in a plastic blend, the fluorescence spectra of dibenzazepine-containing polymers (PAzep-Fls) depended on the compatibilities of the blend. This fluorescent behavior was effective for estimating the compatibility by the fluorescence spectra. The method described in this paper needs only one fluorescent additive; therefore, it is expected that this method of estimating compatibility will be simplified, whereas two kinds of polymers were needed for estimating by using phenazasiline-containing polymers. ${ }^{12}$ Therefore, PAzep-Fls are functional materials for estimating plastic blends. This paper has discussed estimating the compatibility of a PLA/PCL blend, but the versatility of this method will be improved by adapting it to another blend system. This method should be effective for another blend system because not only PAzep-Fl-a (reactive with isocyanate), but also PAzep-Fl-b (non-reactive with isocyanate) was effective 
for estimating the compatibility of the PLA/PCL/LTI blend system. In addition, improvements of the detectability of compatibility by these fluorescent spectra will be an expected measurement method by further molecular design.

\section{Acknowledgements}

The author thanks Prof. Yukio Onouchi (Aichi Institute of Technology: AIT), Mr. Masanobu Maeda (AIT), Mr. Taiki Naruo (AIT), Mr. Hiroki Hirota (AIT), Mr. Junpei Kuno (AIT), Mr. Keiji Murakami (AIT), Mr. Hiroyoshi Inoue (AIT), Dr. Tadashi Ogawa (Aichi Medical University: AMU), Dr. Hidek Hattori (AMU), Prof. Hiroshi Seno (AMU), Dr. Koji Hirano (Nagoya Municipal Industrial Research Institute: nmiri), Dr. Kouji Iida (nmiri), Mr. Masaki Harada (nmiri), Mr. Yuzo Ishigaki (nmiri), and Dr. Mitsuo Oda (nmiri) for their helpful support.

Part of this research was performed under the Cooperative Research Program of "Network Joint Research Center for Materials and Devices". A part of this research was also supported by The Naito Science \& Engineering Foundation, and JKA.

Table 6 Prepared PLA/PCL blend composites and their fluorescence $\lambda_{\max }$

\begin{tabular}{|c|c|c|c|c|c|}
\hline Sample & $\begin{array}{l}\text { PLA, } \\
\text { wt } \%\end{array}$ & $\begin{array}{l}\text { PCL, } \\
\text { wt } \%\end{array}$ & Added PAzep-Fl ${ }^{\mathbf{a}}$ & $\begin{array}{l}\text { LTI/ } \\
\text { phr }\end{array}$ & $\begin{array}{l}\text { Fluorescence } \\
\lambda_{\max } / \mathrm{nm}^{\mathrm{b}}\end{array}$ \\
\hline Blend-a1 & 90 & 10 & PAzep-Fl-a $(0.01)$ & 0 & 426 \\
\hline Blend-a1T & 90 & 10 & PAzep-Fl-a (0.01) & 0.5 & 413 \\
\hline Blend-a2 & 85 & 15 & PAzep-Fl-a $(0.01)$ & 0 & 432 \\
\hline Blend-a2T & 85 & 15 & PAzep-Fl-a $(0.01)$ & 0.5 & 413 \\
\hline Blend-a3 & 70 & 30 & PAzep-Fl-a $(0.01)$ & 0 & 429 \\
\hline Blend-a3T & 70 & 30 & PAzep-Fl-a $(0.01)$ & 0.5 & 415 \\
\hline Blend-b1 & 90 & 10 & PAzep-Fl-b (0.01) & 0 & 436 \\
\hline Blend-b1T & 90 & 10 & PAzep-Fl-b (0.01) & 0.5 & 422 \\
\hline Blend-b2 & 85 & 15 & PAzep-FI-b (0.01) & 0 & 436 \\
\hline Blend-b2T & 85 & 15 & PAzep-Fl-b $(0.01)$ & 0.5 & 426 \\
\hline Blend-b3 & 70 & 30 & PAzep-Fl-b (0.01) & 0 & 436 \\
\hline Blend-b3T & 70 & 30 & PAzep-Fl-b $(0.01)$ & 0.5 & 433 \\
\hline
\end{tabular}

a. The figures in parenthesis show the added ratio (phr). b. Excited at absorption $\lambda_{\max }$ of PAzep-Fl in $\mathrm{CHCl}_{3}$.

\section{Supporting Information}

A detailed explanation of $\mathrm{phr}$ is given. Fluorescence spectra of PAzep-Fl-containing plastic blend composites and the fluorescence spectra of PAzep-Fl in a mixed solvent are shown. This material is available free of charge on the Web at http:// www.jsac.or.jp/analsci/.

\section{References}

1. Y. Ito, T. Shimada, J. Ha, M. Vacha, and H. Sato, J. Polym. Sci., Part A: Polym. Chem., 2006, 44, 4338.

2. X. Kong, A. P. Kulkarni, and S. A. Jenekhe, Macromolecules, 2003, 36, 8992.

3. L. Y. Yang, L. Q. Li, C. Wang, F. J. Zhu, Y. Lu, S. Janietz, A. Wedel, Y. L. Hua, and S. G. Yin, J. Lumin., 2007, 122-123, 714.

4. L. Y. Yang, C. Wang, L. Q. Li, S. Janietz, A. Wedel, Y. L.

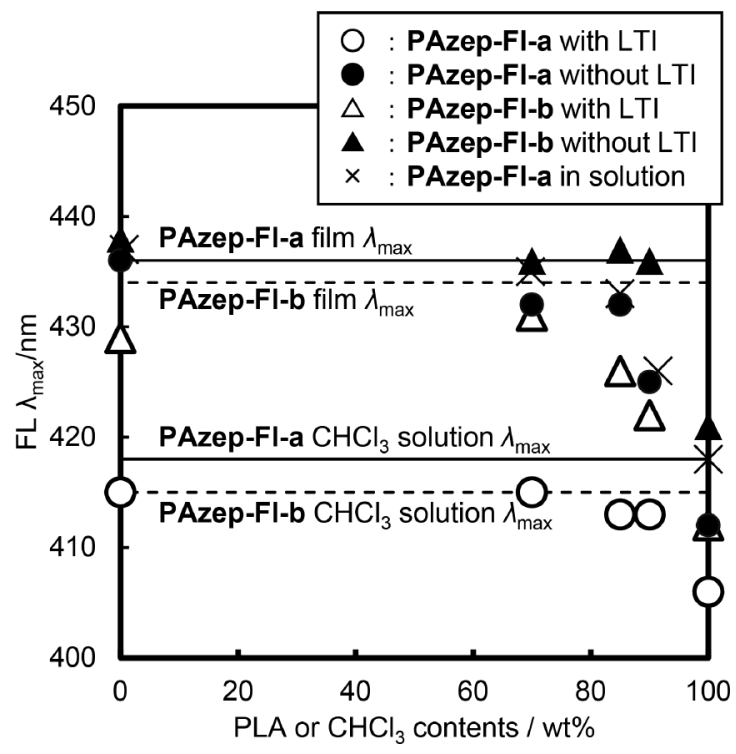

Fig. 14 Relationship between the fluorescence $\lambda_{\max }$ and the weight ratio of a matrix plastic or solvent.

(a) Blend composite with PAzep-FI-a

(b) Blend composite with PAzep-FI-b
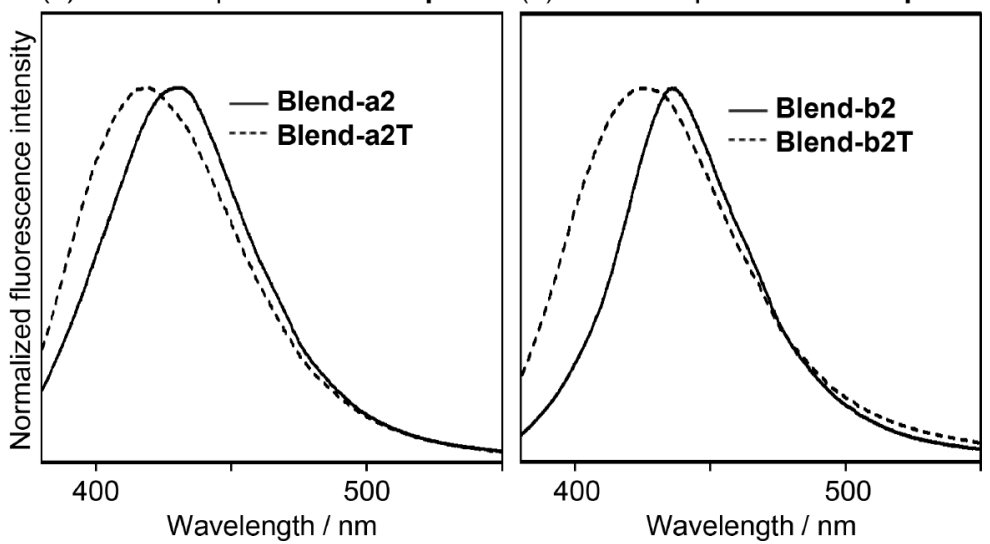

Fig. 13 Fluorescence spectra of PLA:PCL $=85: 15$ blend composite with PAzep-Fls. 
Hua, and S. G. Yin, J. Polym. Sci., Part A: Polym. Chem., 2007, 45, 4291.

5. Y. Chen and T.-Y. Wu, Polymer, 2001, 42, 9895.

6. T.-Y. Wu, R.-B. Sheu, and Y. Chen, Macromolecules, 2004, 37, 725 .

7. H. Kondo, M. Sato, and M. Yokoyama, J. Polym. Sci., Polym. Chem. Ed., 1983, 21, 165.

8. M. Mastragostino, A. Zanelli, G. Casalbore-Miceli, and A. Geri, Synth. Met., 1995, 68, 157.

9. K. Ono, T. Kobayashi, Y. Sato, K. Eguchi, S. Kato, N. Kishi, and T. Soga, Heterocycles, 2011, 83, 1977.

10. W. Lu, J. Kuwabara, T. Iijima, H. Higashimura, H. Hayashi, and T. Kanbara, Macromolecules, 2012, 45, 4128.

11. H. Hayashi, Koubunshi Ronbunshu, 2006, 63, 529.

12. H. Hayashi, H. Nakao, and K. Iida, Bunseki Kagaku, 2011, $60,215$.

13. H. Nakao, H. Hayashi, and K. Okita, Bunseki Kagaku, 2000, 49, 169.

14. H. Hayashi, M. Murase, T.-a. Koizumi, K. Ohara, T. Miyabayashi, and M. Kojima, Bull. Chem. Soc. Jpn., 2010, 83, 1282.

15. H. Hayashi, H. Nakao, T. Miyabayashi, and M. Murase, Jpn. J. Appl. Phys., 2013, 52, 05DA13.

16. H. Hayashi, H. Nakao, A. Adachi, H. Kimura, K. Okita, T. Hayashi, and M. Tanaka, Chem. Lett., 2000, 688.

17. H. Hayashi, H. Nakao, S.-y. Onozawa, A. Adachi, T. Hayashi, and K. Okita, Polym. J., 2003, 35, 704.

18. H. Nakao, H. Hayashi, T. Yoshino, S. Sugiyama, K. Otobe, and T. Ohtani, Nano Lett., 2002, 2, 475.

19. H. Nakao, H. Hayashi, F. Iwata, H. Karasawa, K. Hirano, S. Sugiyama, and T. Ohtani, Langmuir, 2005, 21, 7945.

20. K. Otobe, H. Nakao, H. Hayashi, F. Nihey, M. Yudasaka, and S. Iijima, Nano Lett., 2002, 2, 1157.

21. A. Södergård and M. Stolt, Prog. Polym. Sci., 2002, 27, 1123.

22. S. Taguchi, J. Adhes. Soc. Jpn., 2016, 52, 44.

23. M. Jamshidian, E. A. Tehrany, M. Imran, M, Jacquot, and S. Desobry, Compr. Rev. Food Sci. Food Saf., 2010, 9, 552.

24. T. Li, J. Zhang, D. K. Schneiderman, L. F. Francis, and F. S. Bates, ACS Macro Lett., 2016, 5, 359.

25. L.-F. Wang, J.-W. Rhim, and S.-I. Hong, LWT-Food Sci. Technol., 2016, 68, 454.

26. J. Kylmä and J. V. Seppälä, Macromolecules, 1997, 30, 2876.

27. M. Stolt, K. Hiltunen, and A. Södergård, Biomacromolecules, 2001, 2, 1243.

28. R. F. Storey, J. S. Wiggins, and A. D. Puckett, J. Polym. Sci., Part A: Polym. Chem., 1994, 32, 2345.

29. J. Pan, Y. Wang, S. Qin, B. Zhan, and Y. Luo, J. Biomed. Mater. Res. Part B, 2005, 74, 476.

30. M. Hiljanen-Vainio, P. Varpomaa, J. Seppälä, and P. Törmälä, Macromol. Chem. Phys., 1996, 197, 1503.

31. S. Aslan, L. Calandrelli, P. Laurienzo, M. Malinconico, and C. Migliaresi, J. Mater. Sci.: Mater. Med., 2000, 35, 1615.

32. G. Maglio, M. Malinconico, A. Migliozzi, and G. Groeninckx, Macromol. Chem. Phys., 2004, 205, 946.

33. W. Song, H. Liu, F. Chen, and J. Zhang, Polymer, 2012, 53, 2476.

34. V. Vilay, M. Mariatti, Z. Ahmad, K. Pasomsouk, and M. Todo, Mater. Sci. Eng. A, 2010, 527, 6930.

35. K. Hashima, S. Nishitsuji, and T. Inoue, Polymer, 2010, 51, 3934.

36. H. Tsuji, H. Takai, N. Fukuda, and H. Takikawa, Macromol. Mater. Eng., 2006, 291, 325.

37. N. Fukuda and H. Tsuji, J. Appl. Polym. Sci., 2005, 96, 190.

38. L. Wang, W. Ma, R. A. Gross, and S. P. McCarthy, Polym. Degrad. Stab., 1998, 59, 161.

39. M. Tomita, E. Lavik, H. Klassen, T. Zahir, R. Langer, and M. J. Young, Stem Cells, 2005, 23, 1579.
40. M. Oda, H. Fukuda, M. Harada, and K. Iida, Jpn. Kokai Tokkyo Koho, 2009, 144056.

41. Y. Ishigaki, M. Oda, Y. Takagi, H. Hayashi, M. Yamanaka, Y. Murase, and K. Takahashi, Res. Rep. Nagoya Muni. Ind. Res. Inst., 2016, 101, 20.

42. S. S. Ray, K. Okamoto, and M. Okamoto, Macromolecules, 2003, 36, 2355.

43. Y. Takagi, R. Yasuda, M. Yamaoka, and T. Yamane, J. Appl. Polym. Sci., 2004, 93, 2363.

44. H. Hayashi, T. Yamamoto, M. Sakamoto, K. Iida, H. Nakao, K. Hirano, M. Kawaguchi, T. Hibino, S. Takenaka, and T. Hattori, Polym. J., 2005, 37, 52.

45. H. Hayashi, M. Harada, M. Maeda, and Y. Onouchi, J. Jpn. Soc. Colour Mater., 2018, 91, 13.

46. L. Hearn, R. A. Moore, S. Derry, P. J. Wiffen, and T. Phillips, Cochrane Database of Systematic Reviews, 2014, Article No.: CD010769.

47. L. Hearn, R. A. Moore, S. Derry, P. J. Wiffen, and T. Phillips, Cochrane Database of Systematic Reviews, 2014, Article No.: CD011003.

48. H. Hattori, E. Takashima, T. Yamada, and O. Suzuki, J. Chromatogr., 1990, 529, 189.

49. M. Harada, K. Iida, K. Okamoto, H. Hayashi, and K. Hirano, Polym. Eng. Sci., 2008, 48, 1359.

50. M. Harada, T. Ohya, K. Iida, H. Hayashi, K. Hirano, and H. Fukuda, J. Appl. Polym. Sci., 2007, 106, 1813.

51. H. Hayashi, K. Murakami, H. Inoue, H. Hirota, M. Harada, K. Iida, Y. Onouchi, and K. Hirano, Curr. Trends Polym. Sci., 2010, 14, 11.

52. M. Aiba, Y. Shibasaki, M. Oda, C. Ooka, S. Fukuta, T. Higashihara, S. Ando, M. Ueda, and W.-C. Chen, J. Polym. Sci. Part A: Polym. Chem., 2019, 57, 878.

53. T. Yamamoto and H. Hayashi, J. Polym. Sci., Part A: Polym. Chem., 1997, 35, 463.

54. T. Hagiwara, M. Yamaura, and K. Iwata, Nippon Kagaku Kaishi, 1989, 1791.

55. M. Nomura, Y. Shibasaki, M. Ueda, K. Tsugita, M. Ishikawa, and Y. Taniguchi, Macromolecules, 2004, 37, 1204.

56. H. Hayashi, K. Murakami, Y. Onouchi, M. Oda, and H. Nakao, J. Jpn. Soc. Colour Mater, 2019, 92, 2.

57. H. Hayashi, T. Ogawa, H. Seno, Y. Ishigaki, and M. Oda, Jpn. Soc. Biomed. Mass Spectrom. Lett. Suppl., 2019, 44, 59.

58. T. Yamamoto and T.-a. Koizumi, Polymer, 2007, 48, 5449.

59. S.-B. Kim, K. Harada, and T. Yamamoto, Macromolecules, 1998, 31, 988.

60. T. Yamamoto, S.-B. Kim, and T.-a. Koizumi, Polym. J., 2009, 41, 810 .

61. M. L. Post, O. Kennard, and A. S. Horn, Acta Crystallogr., 1975, B31, 1008.

62. H. Hayashi, Y. Ishigaki, J. Kuno, H. Hirota, T. Ogawa, H. Hattori, H. Seno, Y. Onouchi, and M. Oda, J. Adhes. Soc. Jpn., 2015, 51, 298.

63. H. Hayashi, M. Maeda, T. Naruo, Y. Onouchi, M. Harada, and Y. Ishigaki, Int. J. Polym. Anal. Charact., 2017, 22, 725.

64. H. Hayashi, M. Maeda, J. Kuno, T. Naruo, H. Hirota, M. Harada, K. Iida, Y. Onouchi, and K. Hirano, Anal. Sci., 2016, 32, 1251.

65. Y. Morisaki, N. Wada, and Y. Chujo, Polymer, 2005, 46, 5884.

66. T. Yamamoto, D. Komarudin, M. Arai, B.-L. Lee, H. Suganuma, N. Asakawa, Y. Inoue, K. Kubota, S. Sasaki, T. Fukuda, and H. Matsuda, J. Am. Chem. Soc., 1998, 120, 2047.

67. A. Iida and S. Yamaguchi, Chem. Commun., 2009, 3002.

68. H. Hayashi, T. Naruo, N. Fukuda, M. Harada, M. Futamura, Y. Onouchi, K. Hirano, and T. Miyake, J. Adhes. Soc. Jpn., 2015, 51, 192. 